\title{
Perinatal Risk Factors for Postnatal Weight Loss in Late Preterm Infants
}

\author{
Seong Phil Bae, $\mathrm{MD}^{1}$, Jun Hwan Song, $\mathrm{MD}^{2}$, Won-Ho Hahn, $\mathrm{PhD}^{1}$, Ji Won Koh, $\mathrm{MD}^{2}$, and Ho Kim, $\mathrm{MD}^{2}$ \\ ${ }^{1}$ Department of Pediatrics, Soonchunhyang University Seoul Hospital, Soonchunhyang University College of Medicine, Seoul, Korea \\ ${ }^{2}$ Department of Pediatrics, Soonchunhyang University Cheonan Hospital, Soonchunhyang University College of Medicine, Cheonan, \\ Korea
}

\section{ABSTRACT}

Purpose: Many studies have reported associations of early postnatal growth failure in preterm infants with several morbidities. However, the risk factors for postnatal weight loss (PWL) in late preterm infants have not been identified. We investigated the independent risk factors for PWL in late preterm infants.

Methods: This was a retrospective cohort study. We enrolled 369 late preterm infants born at $34^{+0}$ to $36^{+6}$ weeks gestational age who were admitted to the Soonchunhyang University Cheonan Hospital between 2015 and 2017. PWL\% was calculated as (birth weight-lowest weight $) /$ birth weight $\times 100$. The infants were classified into lower $(<5 \%)$ and higher $(\geq 10 \%)$ PWL\% groups by propensity score matching for gestational age, sex, and birth weight. Perinatal risk factors were analyzed using multivariable logistic regression.

Results: The lower and higher PWL\% groups included 62 and 31 infants, respectively. Antenatal steroids administered within 1 week before birth (odds ratio [OR], 3.26; 95\% confidence interval [CI], 1.015 to 10.465; $P=0.047$ ), lower total calorie intake during days 1 to 7 (OR, 0.98; 95\% CI, 0.977 to $0.999 ; P=0.027$ ), and phototherapy (OR, 5.28; 95\% CI, 1.327 to $21.024 ; P=0.018$ ) were independent risk factors for the higher PWL\%.

Conclusion: Further studies are needed to identify the risk factors that cause high PWL\% according to gestational age and short- and long-term morbidities based on the degree of PWL.

Key Words: Infant, Premature; Weight loss; Betamethasone; Comorbidity

\section{INTRODUCTION}

Early changes in body weight after birth are known to be due to a decline in extracellular fluid and are affected by energy expended to adapt to the extrauterine environment and to survive and grow. Recently, Paul et al. ${ }^{1)}$ reported that early weight change varied according to the mode of delivery and that $5 \%$ to $8 \%$ of infants had not recovered to their birth weight by 3 weeks of age even in term infants.
Received: 22 May 2019

Revised: 8 July 2019

Accepted: 8 July 2019

Correspondence to: Jun Hwan Song, MD

Department of Pediatrics, Soonchunhyang University Cheonan Hospital, Soonchunhyang University College of Medicine, 31 Suncheonhyang 6-gil, Dongnam-gu, Cheonan 31151, Korea Tel: +82-41-570-2160

Fax: +82-41-572-4996

E-mail: joonanii@naver.com
Copyright(c)

By Korean Society of Neonatology.

All right reserved.

This is an Open-Access article distributed under the terms of the Creative Commons Attribution Non-Commercial License (http://creativecommons.org/licenses/ by-nc/4.0), which permits unrestricted non-commercial use, distribution, and reproduction in any medium, provided the original work is properly cited. 
Late preterm infants are relatively immature compared to term infants and are reported to have a higher incidence of morbidity and organ immaturity than term infants ${ }^{2-4)}$. There are limited data with respect to standardized growth curves and nutritional recommendations for the early postnatal period of late preterm infants. Therefore, a consensus on interpretations and interventions to support early weight change is lacking ${ }^{5)}$.

Many studies have investigated the risk factors, clinical outcomes of postnatal weight loss (PWL), and comorbidities associated with early postnatal growth failure in infants with extremely low birth weight or low gestational age ${ }^{6,7)}$. However, few studies have been conducted on the PWL in late preterm infants, which are the most prevalent among preterm infants. Thus, we conducted this study to identify the independent perinatal risk factors affecting the PWL percent (PWL\%) in late preterm infants.

\section{MATERIALS AND METHODS}

\section{Subjects}

This study was a retrospective cohort study that included infants born at $34^{+0}$ to $36^{+6}$ weeks of gestational age who were admitted to the Soonchunhyang Cheonan University Hospital between January 1, 2015, and December 31, 2017. Infants were excluded from the study if they had congenital anomalies, were transferred to another hospital, received total parenteral nutri tion (TPN), were under nothing by mouth (nil per os [NPO]) for longer than 24 hours, or lacked a complete medical record. Perinatal and clinical course data were collected retrospectively from the medical records. PWL\% was calculated as (birth weightlowest weight)/birth weight $\times 100$. The infants were classified into lower $(<5 \%)$ and higher $(\geq 10 \%)$ PWL\% groups by propensity score matching for gestational age, sex, and birth weight.

\section{Care environment}

All late preterm infants were admitted to the neonatal inten sive care unit (NICU), received care in incubators until the 5th day of life, and were maintained at a similar temperature $\left(32.8^{\circ} \mathrm{C}\right.$ to $33.8^{\circ} \mathrm{C}$ ) and humidity ( $60 \%$ to $\left.65 \%\right)$. The incubator care was tapered according to our NICU protocol. When the infant's weight steadily increased and feeding progressed well, incubator care was stopped and light-emitting diode (LED) phototherapy was initiated if jaundice was detected (Natus Medical, München, Germany).

\section{Fluids}

If the infant was healthy, feeding was initiated on the first day of life. If the infant's general condition was not good or feeding was not progressing well, additional intravascular fluid was administered instead of full oral feeding. If there was insufficient feeding during the first 5 days, following the protocol of the NICU, the feeding was increased to $60 \mathrm{~mL} / \mathrm{kg}$ on day $1,80 \mathrm{~mL} / \mathrm{kg}$ on day 2, $100 \mathrm{~mL} / \mathrm{kg}$ on day 3, $120 \mathrm{~mL} / \mathrm{kg}$ on day 4, and $150 \mathrm{~mL} /$ $\mathrm{kg}$ on day 5. Until the infants recovered their birth weight, the birth weight was used to calculate feeding amounts. The deficit amount of feeding was supplemented by intravascular administration of $10 \%$ to $12.5 \%$ dextrose water.

\section{Statistical analyses}

We collected infant demographics and data on maternal factors, comorbidities, and hospital courses for univariable analysis of the different PWL\% groups. Categorical data were analyzed using a chi-square test, and the Mann-Whitney test was performed for continuous data that were not distributed normally. Variables with a significance of $P \leq 0.2$ in the univariable analysis were analyzed by multivariable logistic regression analysis with the stepwise method and significance was set at $P<0.05$.

Statistical analyses were performed using SPSS version 25.0 (IBM Corp., Armonk, NY, USA) and R package version v3.3.1 (R Foundation for Statistical Computing, Vienna, Austria).

\section{RESULTS}

Of the 469 infants born during the study period, 369 were

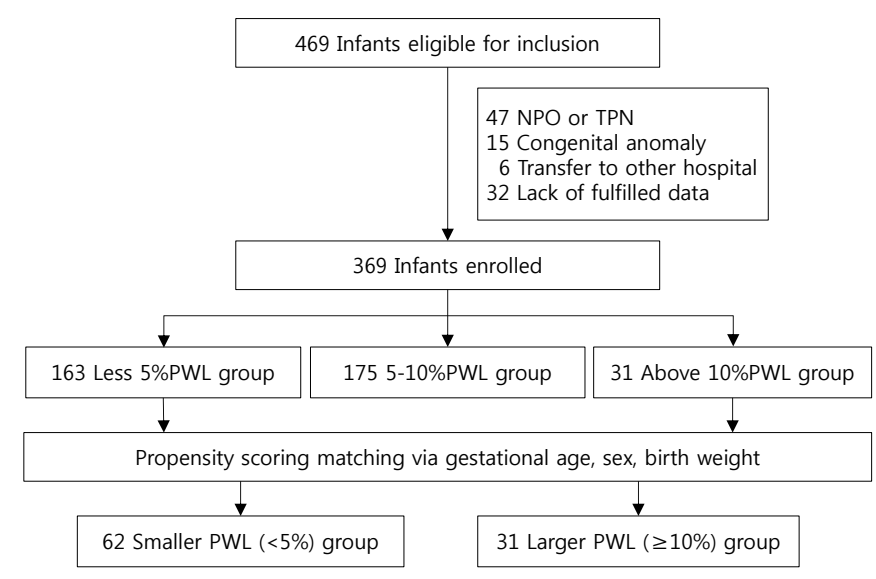

Figure 1. Flow chart of study population. Abbreviations: NPO, nil per os; TPN, total parenteral nutrition; PWL, postnatal weight loss. 
included in the study. Forty-seven infants under NPO restrictions for longer than 24 hours or TPN, 15 with congenital anomalies, six transferred to other hospitals, and 32 infants lacking complete data were excluded (Figure 1). According to propensity score matching for gestational age, sex, and birth weight, infants were classified into the lower ( $n=62)$ and higher $(n=31)$ PWL\% groups.

Analysis of maternal factors revealed that the administration of antenatal steroids (ANS) within 1 week before birth $(P<0.001)$ and gestational diabetes mellitus $(P=0.026)$ differed significantly between the groups. For the ANS, betamethasone was administered in all but three of the infants receiving dexamethasone. There were no significant differences in the other maternal fac tors analyzed (Table 1).

There were no significant differences in birth height or head circumference between the groups. The small for gestational age or with birth weight $Z$ scores $<-2$ standard deviations, calculated

Table 1. Demographics of Infants, Maternal Characteristics, Comorbidities, and Hospital Courses of Lower and Higher Postnatal Weight Loss Groups

\begin{tabular}{|c|c|c|c|}
\hline Variable & Smaller PWL group $(n=62)$ & Larger PWL group $(n=31)$ & $P$-value \\
\hline \multicolumn{4}{|l|}{ Demographics of infants } \\
\hline GA (wk) & $35(34.4-35.4)$ & $35.1(34.2-35.5)$ & 0.719 \\
\hline $\mathrm{BH}$ & $46.75(45-48)$ & $46(44.5-48)$ & 0.422 \\
\hline BW z score $<-2$ SD & $9(14.5)$ & $7(22.6)$ & 0.331 \\
\hline \multicolumn{4}{|l|}{ Maternal characteristics } \\
\hline Maternal age (yr) & $32(29-35)$ & $32(31-36)$ & 0.239 \\
\hline Antenatal steroid within $1 \mathrm{wk}$ before birth & $15(24.2)$ & $19(61.3)$ & $<0.001$ \\
\hline IVF & $9(14.5)$ & $7(22.6)$ & 0.331 \\
\hline Maternal thyroid disease & $19(10.3)$ & $15(8.1)$ & 0.461 \\
\hline \multicolumn{4}{|l|}{ Comorbidities } \\
\hline Apgar score $1 \mathrm{~min}$ & $6.5(6-7.25)$ & $7(5-8)$ & 0.748 \\
\hline Apgar score 5 min & $8(8-9)$ & $9(8-10)$ & 0.312 \\
\hline Surfactant & $4(6.5)$ & $2(6.5)$ & 1.000 \\
\hline Mechanical ventilation & $2(3.2)$ & $2(6.5)$ & 0.598 \\
\hline TTN & $6(9.7)$ & 0 & 0.173 \\
\hline Oxygen therapy & $16(25.8)$ & $6(19.4)$ & 0.490 \\
\hline Phototherapy & $23(37.1)$ & $26(83.9)$ & $<0.001$ \\
\hline IVH & $5(8.2)$ & $3(9.7)$ & 1.000 \\
\hline Total hospitalization period (d) & $16.5(12.25-20)$ & $19(16-24.5)$ & 0.021 \\
\hline Recover to birth weight at discharge & $30(48.4)$ & $1(3.2)$ & $<0.001$ \\
\hline
\end{tabular}

Values are expressed as median (interquartile range) or number (\%).

Abbreviations: PWL, postnatal weight loss; GA, gestational age; BW; birth weight; BH, birth height; IVF, in vitro fertilization; PPROM, preterm premature rupture of membranes; GDM, gestational diabetes mellitus; PIH, pregnancy induced hypertension; TTN, transient tachypnea of newborn; IVH, intraventricular hemorrhage. 
by the INTERGROWTH-21st standard, was not significantly different between the different groups.

The phototherapy was significantly higher in the higher PWL\% group than in the lower PWL\% group $(P<0.001)$. Regarding the hospital course, total fluid intake $(P=0.005)$ and total calorie intake $(P=0.002)$ during day 1 to 7 were significantly lower and the time to achieve full feeding $(100 \mathrm{~mL} / \mathrm{kg} / \mathrm{day}, P<0.001)$ was longer in the higher PWL\% group than in the lower PWL\% group. The higher PWL\% group had a significantly longer hospitalization period $(P=0.021)$, and the majority of infants in the higher PWL\% group were discharged without recovering to their birth weight $(P<0.001)$

Regarding perinatal risk factors, the multivariate logistic regression analysis revealed that administration of ANS within 1 week before birth (odds ratio [OR], 3.26; 95\% confidence interval [CI], 1.015 to $10.465 ; P=0.047$ ) was found to increase the risk of a higher PWL\%. Lower total calorie intake during day 1 to 7 (OR, 0.98; 95\% $\mathrm{CI}, 0.977$ to $0.999 ; P=0.027)$ and phototherapy (OR, 5.28; 95\% CI, 1.327 to 21.024; $P=0.018$ ) were identified as independent risk factors for a higher PWL\% (Table 2).

\section{DISCUSSION}

The water content varies according to gestational age and even varies in infants of the same gestational age ${ }^{8)}$. Preterm infants require more energy than term infants to achieve optimal intrau terine weight gains, but their energy expenditure is higher owing to comorbidities, difficulties with nutritional support, and the immaturity of multiple organs. Therefore, it is difficult to judge the proper PWL according to gestational age or birth weight.

This study was performed to investigate the risk factors affect-

Table 2. Multivariable Logistic Regression Analysis of Perinatal Risk Factors for the Higher Postnatal Weight Loss Group

\begin{tabular}{lcc}
\hline Variable & Adjust OR (CI) & $P$-value \\
\hline $\begin{array}{l}\text { Antenatal steroid within 1 wk before } \\
\text { birth }\end{array}$ & $3.26(1.015-10.465)$ & 0.047 \\
$\begin{array}{l}\text { Total calorie intake during day 1-7 } \\
\text { (kcal/kg) }\end{array}$ & $0.98(0.977-0.999)$ & 0.027 \\
Phototherapy & $5.28(1.327-21.024)$ & 0.018
\end{tabular}

Adjusted for gestational diabetes mellitus, antenatal steroids, phototherapy, total fluid intake during day $1-5$, total calorie intake during day $1-5$.

Abbreviations: OR, odds ratio; $\mathrm{CI}$, confidence interval. ing the PWL\% in late preterm infants. The administration of ANS within 1 week before birth, lower total calorie intake during day 1 to 7 after birth, and phototherapy were identified as independent perinatal risk factors influencing the PWL\%. However, postnatal comorbidities were not found to affect the PWL\%.

Interestingly, ANS administration was found to affect PWL\% in the present study. ANS have many complex actions that have short-term and long-term effects ${ }^{9-11}$. In infants with extremely low birth weight, ANS had been reported to decrease PWL by reducing insensible water loss and promoting earlier diuresis and natriuresis ${ }^{12,13)}$. Conversely, in this study of late preterm infants, ANS was reported to be an independent risk factor for increased PWL\%. A recent study suggested that ANS might modify the metabolic state by inducing hormonal changes in late preterm infants ${ }^{14)}$. Another study reported a higher prevalence of hypoglycemia in late preterm infants who received ANS ${ }^{15}$.

Growth patterns of preterm infants have been reported. Thorp et al. ${ }^{16)}$ reported that ANS did not affect weight gain in preterm infants. Battin et al. ${ }^{17)}$ reported that repeated ANS administration affected postnatal growth acceleration 3 to 5 weeks after birth. However, studies on growth pattern changes in late preterm infants are rare. Additional studies will be needed on growth patterns and prognoses according to antenatal ANS dosing.

The total calorie intake for the first week of life in the higher PWL\% group was lower than that of the lower PWL\% group in the current study. Because the infants enrolled in this study did not receive an intravenous amino acid solution or a lipid formulation, this difference in caloric value was thought to be due to delayed full feeding. Delayed full feeding was reported to be one of the important factors of postnatal growth failure in infants with very low birth weight, and enhanced feeding was shown to reduce growth failure ${ }^{18,19)}$. However, feeding progression is influenced not only by neurological development but also by various other factors such as medical conditions, milk types, and feeding schedules ${ }^{20,21)}$. Thus, the authors concluded that if feeding progression is slow and a mother had a history of ANS administration, close monitoring and nutritional supplementation might be necessary in late preterm infants.

The association between the degree of PWL and the clinical outcome of premature infants has not been clear, ${ }^{6,12,22)}$. However, in term infants, excessive weight loss was reported to increase the risk of hypernatremia, dehydration, and readmission for hyperbilirubinemia ${ }^{23,24)}$. In infants with extremely low birth weight, postnatal early weight loss had also been reported to 
reduce the risk of bronchopulmonary dysplasia or death ${ }^{25,26)}$. In this study, the higher PWL\% group underwent more phototherapy than the lower PWL\% group. All infants received incubator care until the fifth day of life and LED phototherapy, which has little effect on transepidermal water loss ${ }^{27)}$. In the higher PWL\% group, total calorie intake was significantly lower than that in the lower PWL\% group, and weight loss is also known to cause hyperbilirubinemia. Therefore, if hyperbilirubinemia is observed in the PWL state, the overall nutritional status and insensible water loss should be considered.

Most of the late preterm infants had shorter hospital stay to monitor growth unless there were complications. Regarding the hospital course, the higher PWL\% group had a long hospitalization period, and most of the infants in this group had not reco vered their birth weight at discharge.

Further studies are needed to identify risk factors for a high PWL\% according to each gestational age and to establish stan dard comparators to assess the early weight change of late preterm infants for prompt and proper intervention.

To our knowledge, this is the first report that showed the effect of ANS on PWL in late preterm infants. However, this study had several limitations. First, the exact total fluid intake, urinary volume, and insensible water loss could not be measured as this was a retrospective study. Second, the composition of the fluid therapy differed slightly between infants. Third, some infants were discharged without recovering to their birth weight. Therefore, we did not know the rate of recovery to birth weight or growth rate for these infants.

In conclusion, administration of ANS within 1 week before birth, lower total calorie intake during day 1 to 7 , and photo. therapy were independent risk factors for a greater PWL\%. It is necessary to evaluate the degree of appropriate PWL according to gestational age and to conduct further studies on the shortand long-term morbidities according to the degree of PWL.

\section{ARTICLE INFORMATION}

\section{Ethical statement}

This study was approved by the Institutional Review Board of Soonchunhyang University Cheonan Hospital (SCHCA 2019-04020). Written informed consent by the patients was waived due to a retrospective nature of our study.

\section{Conflicts of interest}

No potential conflict of interest relevant to this article was reported.

\section{Author contributions}

Conception or design: S.P.B.

Acquisition, analysis, or interpretation of data: S.P.B., J.W.K., H.K.

Drafting the work or revising: W.H.H., J.H.S

Final approval of the manuscript: J.H.S.

\section{ORCID}

Seong Phil Bae https://orcid.org/0000-0002-6447-418X

Jun Hwan Song https://orcid.org/0000-0002-9330-9140

\section{Acknowledgments}

None

\section{REFERENCES}

1. Paul IM, Schaefer EW, Miller JR, Kuzniewicz MW, Li SX, Walsh EM, et al. Weight change nomograms for the first month after birth. Pediatrics 2016;138:e20162625.

2. Jaiswal A, Murki S, Gaddam P, Reddy A. Early neonatal mor bidities in late preterm infants. Indian Pediatr 2011;48:607-11.

3. Teune MJ, Bakhuizen S, Gyamfi Bannerman C, Opmeer BC, van Kaam AH, van Wassenaer AG, et al. A systematic review of se vere morbidity in infants born late preterm. Am J Obstet Gynecol 2011;205:374.

4. Tsai ML, Lien R, Chiang MC, Hsu JF, Fu RH, Chu SM, et al. Prevalence and morbidity of late preterm infants: current status in a medical center of Northern Taiwan. Pediatr Neonatol 2012;53: 171-7.

5. Muelbert M, Harding JE, Bloomfield FH. Nutritional policies for late preterm and early term infants: can we do better? Semin Fetal Neonatal Med 2019;24:43-7.

6. Aksoy HT, Guzoglu N, Eras Z, Gokce IK, Canpolat FE, Uras N, et al. The association of early postnatal weight loss with outcome in extremely low birth weight infants. Pediatr Neonatol 2019; 60:192-6.

7. Bauer K, Versmold H. Postnatal weight loss in preterm neonates less than $1,500 \mathrm{~g}$ is due to isotonic dehydration of the extracellular volume. Acta Paediatr Scand Suppl 1989;360:37-42.

8. Hartnoll G, Betremieux P, Modi N. Body water content of ex tremely preterm infants at birth. Arch Dis Child Fetal Neonatal 
Ed 2000;83:F56-9.

9. Eriksson L, Haglund B, Ewald U, Odlind V, Kieler H. Short and long-term effects of antenatal corticosteroids assessed in a cohort of 7,827 children born preterm. Acta Obstet Gynecol Scand 2009;88:933-8.

10. Mulder EJ, de Heus R, Visser GH. Antenatal corticosteroid therapy: short-term effects on fetal behaviour and haemodynamics. Semin Fetal Neonatal Med 2009;14:151-6.

11. Wapner RJ, Sorokin Y, Mele L, Johnson F, Dudley DJ, Spong CY, et al. Long-term outcomes after repeat doses of antenatal corticosteroids. N Engl J Med 2007;357:1190-8.

12. Verma RP, Shibli S, Fang H, Komaroff E. Clinical determinants and utility of early postnatal maximum weight loss in fluid management of extremely low birth weight infants. Early Hum Dev 2009;85:59-64.

13. Omar SA, DeCristofaro JD, Agarwal BI, La Gamma EF. Effects of prenatal steroids on water and sodium homeostasis in extremely low birth weight neonates. Pediatrics 1999;104(3 Pt 1): 482-8.

14. Sifianou P, Thanou V, Karga H. Metabolic and hormonal effects of antenatal betamethasone after 35 weeks of gestation. J Pediatr Pharmacol Ther 2015;20:138-43.

15. Gyamfi-Bannerman C, Thom EA, Blackwell SC, Tita AT, Reddy UM, Saade GR, et al. Antenatal betamethasone for women at risk for late preterm delivery. N Engl J Med 2016;374:1311-20.

16. Thorp JA, Jones PG, Peabody JL, Knox E, Clark RH. Effect of antenatal and postnatal corticosteroid therapy on weight gain and head circumference growth in the nursery. Obstet Gynecol 2002;99:109-15.

17. Battin M, Bevan C, Harding J. Growth in the neonatal period after repeat courses of antenatal corticosteroids: data from the ACTORDS randomised trial. Arch Dis Child Fetal Neonatal Ed 2012;97:F99-105.
18. Moltu SJ, Blakstad EW, Strommen K, Almaas AN, Nakstad B, Ronnestad A, et al. Enhanced feeding and diminished postnatal growth failure in very-low-birth-weight infants. J Pediatr Gastroenterol Nutr 2014;58:344-51.

19. Lee SM, Kim N, Namgung R, Park M, Park K, Jeon J. Prediction of postnatal growth failure among very low birth weight infants. Sci Rep 2018;8:3729.

20. Binchy A, Moore Z, Patton D. Feeding intervals in premature infants $\leq 1750$ g: an integrative review. Adv Neonatal Care 2018; 18:168-78.

21. Li YW, Yan CY, Yang L, Han ZL. Effect of breastfeeding versus formula milk feeding on preterm infants in the neonatal intensive care unit. Zhongguo Dang Dai Er Ke Za Zhi 2017;19:572-5.

22. Verma RP, Shibli S, Komaroff E. Postnatal transitional weight loss and adverse outcomes in extremely premature neonates. Pediatr Rep 2017;9:6962.

23. Modi N. Clinical implications of postnatal alterations in body water distribution. Semin Neonatol 2003;8:301-6.

24. Salas AA, Salazar J, Burgoa CV, De-Villegas CA, Quevedo V, Soliz A. Significant weight loss in breastfed term infants readmitted for hyperbilirubinemia. BMC Pediatr 2009;9:82.

25. Wadhawan R, Oh W, Perritt R, LaptookAR, Poole K, Wright LL, et al. Association between early postnatal weight loss and death or BPD in small and appropriate for gestational age extremely low-birth-weight infants. J Perinatol 2007;27:359-64.

26. Oh W, Poindexter BB, Perritt R, Lemons JA, Bauer CR, Ehrenkranz RA, et al. Association between fluid intake and weight loss during the first ten days of life and risk of bronchopulmonary dysplasia in extremely low birth weight infants. J Pediatr 2005;147:786-90.

27. Bertini G, Perugi S, Elia S, Pratesi S, Dani C, Rubaltelli FF. Transepidermal water loss and cerebral hemodynamics in preterm infants: conventional versus LED phototherapy. Eur J Pediatr 2008;167:37-42. 